\title{
Editorial
}

\section{IMRT: Is it superior to formal radiotherapy in the treatment of head neck cancer?}

\begin{abstract}
Introduction:
Incidence of head and neck cancer is increasing day by day all over the world including Bangladesh. Organ preservation rather than organ sacrifice is more preferable and in this context radiotherapy gradually superseded surgery over the last two decades.
\end{abstract}

During the last decade Intensity-modulated radiation therapy (IMRT) represents a major evolution in the treatment of head-and-neck cancer, enhancing the therapeutic ratio by increasing the dose delivered to the tumoral volume while reducing that to healthy organs.

In head-and-neck cancer, it is particularly difficult to ensure a good therapeutic ratio, because:

- Tumors are often large and/or poorly radiosensitive, requiring high therapeutic doses, equal to or greater than $70 \mathrm{~Gy}$ delivered in 35 fractions over 7 weeks for macroscopic lesions and equal to or greater than 50 Gy delivered in 25 fractions over 5 weeks for microscopic lesions.

- Lesion configuration is often ballistically complex, significantly different from simple geometric forms and

- Many healthy at-risk organs are present within the target areas.

\section{History:}

From conformal 3D RT to intensitymodulated radiation therapy:

The evolution in RT techniques over the last 30 years began from $2 \mathrm{D}$ radiation therapy using coplanar beams, usually in opposing pairs: e.g., right and left lateral in head-andneck oncology. Dose distribution was calculated without taking account of tissue heterogeneity, simply according to the skin contour of the target volume. Interposing lead caches here and there in the field helped protect sensitive areas, in what was an early attempt at "conformal" RT.

3D RT was developed using CT data, to determine both the anatomic contours of tissues and organs and radiologic density differentials directly applicable in dosimetrics. As CT data were obtained in all slice planes, 3D reconstruction was possible both for tumor volume and extension and neighboring healthy tissue, and for the dose distribution achieved by the selected treatment protocol. Interposing protection within the beam and, later, using the multileaf collimators available in modern linear accelerators made 3D conformal RT the standard technique by the turn of the century.

IMRT is an evolution of the above technique,The sharp dose fall-off gradient of this technique permits the administration of a highly conformal and more homogeneous dose to the planning target volume (PTV) than conventional and conformal radiotherapy. This allows better sparing of the organs at risk (e.g. parotid glands, submandibular and minor salivary glands, larynx and swallowing structures), leading to a decrease in acute and late side effects.

This may open a window for treatment intensification of radiotherapy alone or 
combined with chemotherapy and/or targeted therapy.In addition, the technique allows dose-escalation, with a higher dose per session delivered to the macroscopic tumor than to other irradiated areas.

\section{Technique}

IMRT uses linear accelerators equipped with multi-leaf collimators with leaf movement controlled by dedicated software. Schematically, two technical solutions are available:

1) A succession of usually five to seven fixed accelerator positions around a circle or arc; so-called "step and shoot" radiation is thus delivered at each position, with intensity modulated by interposition of the collimator leaves;

2) Radiation delivered during rotation through an arc, with continuous intensity modulation, known as "dynamic IMRT" (VMat, Elekta; Rapid'Arc, Varian), which significantly reduces exposure time.

Rationale for intensity-modulated radiation therapy in head-and-neck cancer

IMRT provides three combinable advantages in treatment:

- Relative conservation of healthy tissue;

- Improved tumor coverage;

- And escalation of the dose delivered to the tumor.

Superiority of IMRT over formal radiotherapy in head neck cancer:

Despite of it's efficacy and advantage, the question about it's superiority was still hard to prove. A prospective randomised study, without concurrent chemotherapy, done by Kam et al showed significantly less observerrated severe xerostomia and a significantly higher stimulated parotid and whole saliva flow rate after IMRT treatment for early stage nasopharyngeal carcinoma than twodimensional radiotherapy. Very recent, the first phase IIIrandomized controlled trial of head neck cancer patients done by Nutting et al showed significantly less Grade 2 or more xerostomia at 12 and at 18 months in the IMRT arm than the conventional radiotherapy arm, both without concurrent chemotherapy. No differences in acute mucositis or pain scores were found, although the IMRT group suffered from significantly more acute fatigue of Grade 2 or more.

However, a clear survival benefit of IMRT over the more classic three-dimensional conformal radiation therapy has not been proved as yet, and there are some concerns about the theoretically higher risk of induction of secondary cancers by IMRT because of the increased low-dose irradiated volume. IMRT might also lead to unexpected higher toxicity in areas that were not in the classic two-dimensional beam path but that are irradiated in the IMRT set-up, especially in combination with concurrent chemotherapy.

\section{Conclusion:}

Although the positive impact of this technique on tumor control remains to be proven, salivary function conservation currently makes IMRT the standard treatment in most head-and-neck cancer.

\section{Professor M A Matin}

FRCS (Eng, Edin \& Glasgow)

Professor \& Head

Department of ENT \& HNS

Shaheed Suhrawardy Medical College

Sher-E-Bangla Nagar, Dhaka.

E-mail: matinfrcs@yahoo.com 